\title{
Chapter 25 \\ Broadening the Genetic Diversity of Common and Durum Wheat for Abiotic Stress Tolerance Breeding
}

\author{
Hisashi Tsujimoto, Quahir Sohail, and Yoshihiro Matsuoka
}

\begin{abstract}
An increase in cereal production is required if we are to support a world population of more than nine billion people expected in 2060. For this purpose, plant breeding will serve as a key technology as it did during the Green Revolution of the 1960s. However, the changing climate and decrease in agricultural resources are new challenges that will require consideration. We developed common and durum wheat populations expressing the intraspecific diversity of wild species. We collectively named these populations 'multiple derivative lines', and specifically 'multiple synthetic derivatives' (MSD) in the case of common wheat. The germplasm in the MSD population shows diverse morphology in regular genetic background enabling accurate selection of desired genotypes/phenotypes associated with abiotic stress tolerance. Accordingly, such plant materials can subsequently be used for various breeding purposes. Abiotic tolerance, especially drought tolerance, is largely determined by the interaction between genotype and environment, making drought-tolerance breeding difficult. Wild species, even the self-pollinating ones, possess high diversity which allows them to adapt to the diverse and fluctuating environments. Accordingly, during wheat breeding for abiotic tolerance, it may be better to consider using diverse populations such as the MSD.
\end{abstract}

Keywords Abiotic stress $\bullet$ Aegilops tauschii $\bullet$ Germplasm enhancement $\bullet$ Synthetic wheat $\bullet$ Wheat

\footnotetext{
H. Tsujimoto $(\square)$

Arid Land Research Center, Tottori University, Hamasaka, Tottori 680-0001, Japan e-mail: tsujim@alrc.tottori-u.ac.jp

Q. Sohail

Arid Land Research Center, Tottori University, Hamasaka, Tottori 680-0001, Japan

Biodiversity and Integrated Gene Management Program, International Center for Agricultural Research in the Dry Areas (ICARDA), PO Box 39,

Emek, 06511 Ankara, Turkey

Y. Matsuoka

Fukui Prefectural University, Eiheiji, Yoshida, Fukui 910-1195, Japan
} 
In the next 50 years, an increasing world population will require cereal production to double under radically changing climate conditions. Plant breeding, which played an essential role in the Green Revolution of the 1960s, will serve as a key technology in addressing this problem. However, wheat breeding is becoming increasingly difficult due to the limited genetic variation in the wheat gene pool. This narrow genetic variation is in part a consequence of a bottleneck effect that occurred through very few interspecific hybridization events during the origin of common wheat (Triticum aestivum L., $2 \mathrm{n}=6 \mathrm{x}=42$, AABBDD) 8,000 years ago. Novel alleles can be introduced by crossing with wild relatives; however, the difference in ploidy and morphology between wild and common wheat often hinders proper evaluation of traits, particularly quantitative ones. To date, examples of the successful use of genes or alleles derived from wild species have mostly been limited to qualitative traits such as disease and insect resistance. Here, we aim to produce breeding populations containing the intraspecific variation of the wild species Aegilops tauschii Coss. $(2 \mathrm{n}=2 \mathrm{x}=14, \mathrm{DD})$ and Triticum dicoccoides Koern. $(2 \mathrm{n}=4 \mathrm{x}=28$, AABB $)$ in the genetic background of common and durum wheat varieties. It is hoped that these populations will be useful initial materials during the selection of quantitatively controlled traits such as abiotic stress tolerance.

To develop a breeding population with the genetic background of common wheat, the genetic diversity of 81 Ae. tauschii accessions collected from a range of natural habitats was analyzed using 4,449 polymorphic diversity array technology (DArT) markers (Fig. 25.1; Sohail et al. 2012). The results identified three intraspe-

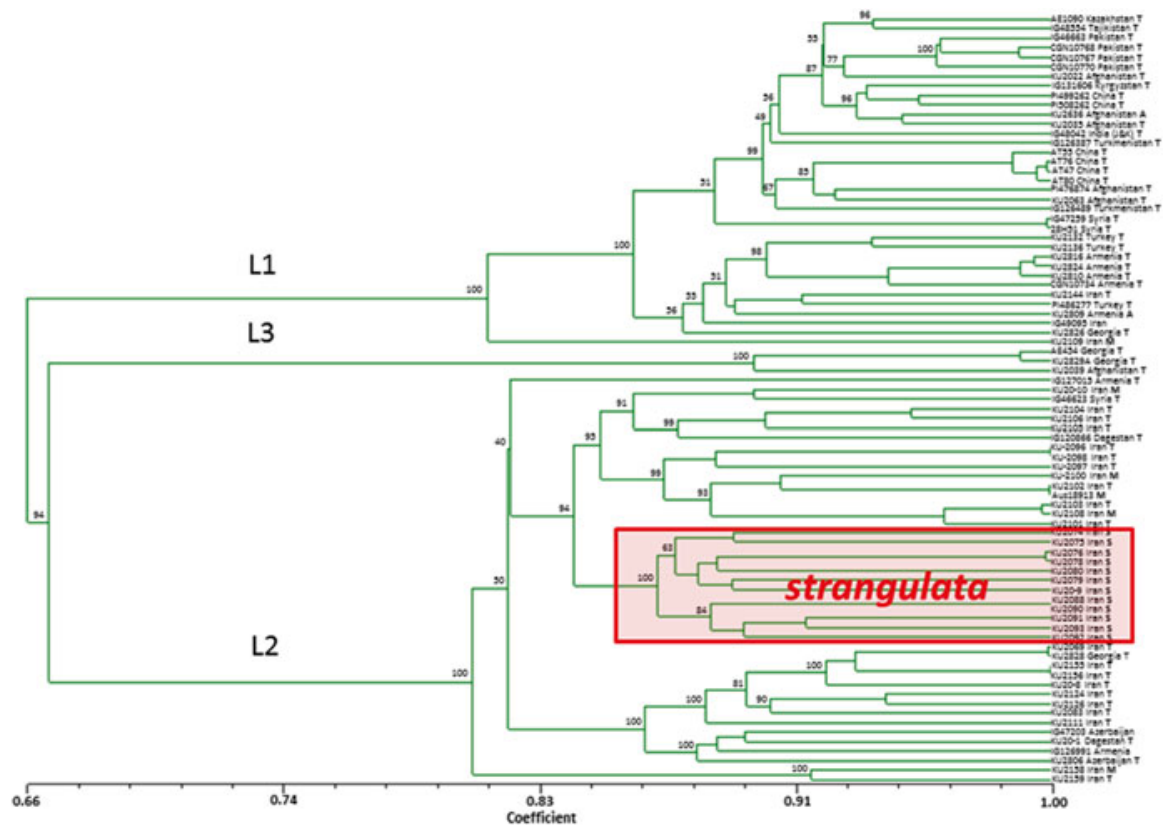

Fig. 25.1 Phylogenetic tree of the 81 accessions of Ae. tauschii constructed using 4,449 DArT markers. Lineages 1, 2 and 3 are indicated by L1, L2 and L3, respectively. The clade of Ae. tauschii ssp. strangulata is boxed 


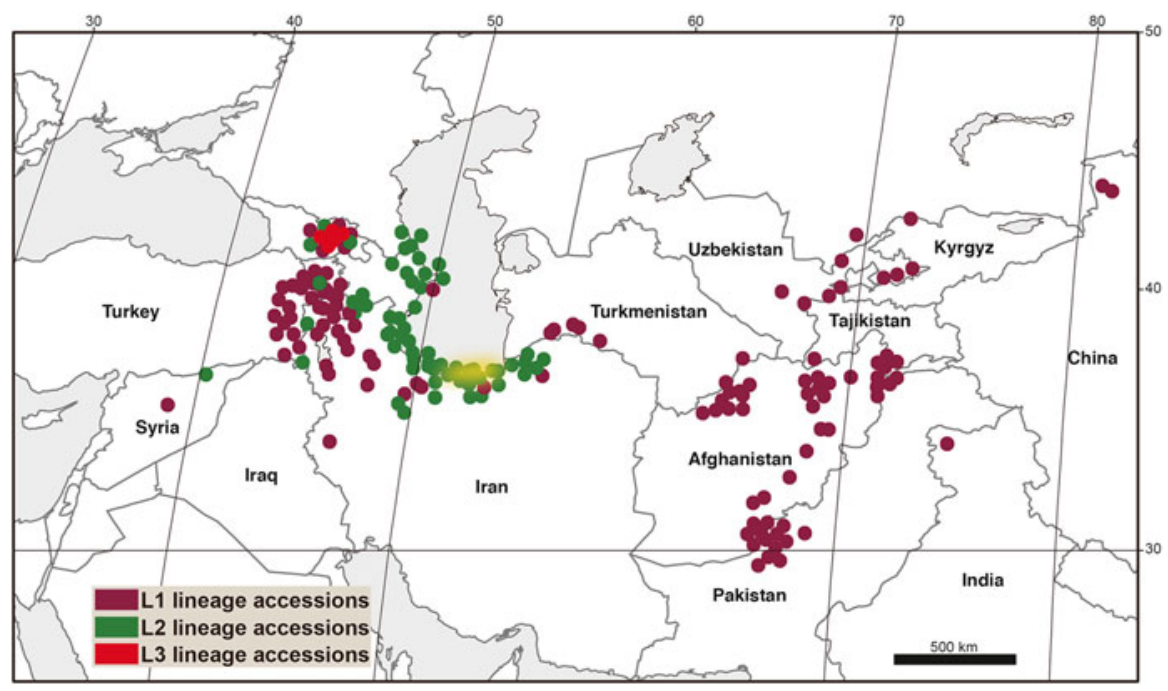

Fig. 25.2 Distribution of Ae. tauschii accessions belonging to Lineages 1, 2 and 3. The habitat area of subspecies strangulata is shaded in yellow

cific lineages: Lineage 1, distributed widely in Central and Western Asia; Lineage 2, distributed in Transcaucasia and northern Iran; and Lineage 3, found in a specific region of Armenia (Fig. 25.2). Because Lineage 2, which includes Ae. tauschii ssp. strangulata, has a close genetic relationship with the $\mathrm{D}$ genome of $T$. aestivum relative to Lineage 1 (Matsuoka et al. 2013), common wheat may show reduced genetic variation compared to the overall interspecific diversity of this species. Fifty-one of the 81 accessions were then randomly selected and crossed with durum wheat (Triticum durum Desf., $2 \mathrm{n}=4 \mathrm{x}=28$, AABB), cv. 'Langdon', to produce 51 amphidiploids, designated 'primary synthetics' or 'synthetic hexaploid wheat' (SHW).

The drought tolerance-related traits of these SHWs were then examined together with those of the original Ae. tauschii accessions under well-watered and drought conditions. The growth performance of the parental Ae. tauschii accessions and their derivative SHWs was then compared (Table 25.1; Sohail et al. 2011). Under well-watered conditions, intercellular $\mathrm{CO}_{2}$, transpiration rate, root dry weight and water use efficiency were significantly correlated in both diploid and hexaploid plants. Under drought conditions, however, no correlation was observed, indicating that the degree of drought tolerance cannot be predicted by the performance of the wild species. In general, wild species have specific mechanisms that allow them to cope with environmental stresses, but if such mechanisms are not transferable to common wheat, they cannot be utilized in breeding programs. The expression of drought-tolerance traits must therefore be evaluated in hexaploid wheat. In contrast, traits that are controlled by a small number of major genes could be screened first using accessions of the wild species. Furthermore, the SHW lines were often unsuitable for evaluation of drought tolerance, because they usually presented a 
Table 25.1 Correlation between the morphological and physiological traits of the Ae. tauschii accessions and their derivative SHW lines under well-watered and drought conditions (Sohail et al. 2011, modified)

\begin{tabular}{l|l|c}
\hline Trait & Well-watered conditions & Drought conditions \\
\hline Photosynthesis & 0.09 & 0.02 \\
\hline Stomata conductance & 0.15 & -0.11 \\
\hline Intercellular $\mathrm{CO}_{2}$ & $0.49^{* *}$ & 0.32 \\
\hline Transpiration rate & $0.37^{*}$ & 0.05 \\
\hline Chlorophyll content & 0.07 & 0.34 \\
\hline Leaf water potential & -0.24 & 0.20 \\
\hline Root dry weight & $0.36^{*}$ & 0.30 \\
\hline Shoot dry weight & 0.25 & 0.15 \\
\hline Water use efficiency & $0.36^{*}$ & 0.24 \\
\hline
\end{tabular}

$* * P<0.01 ; * P<0.05$

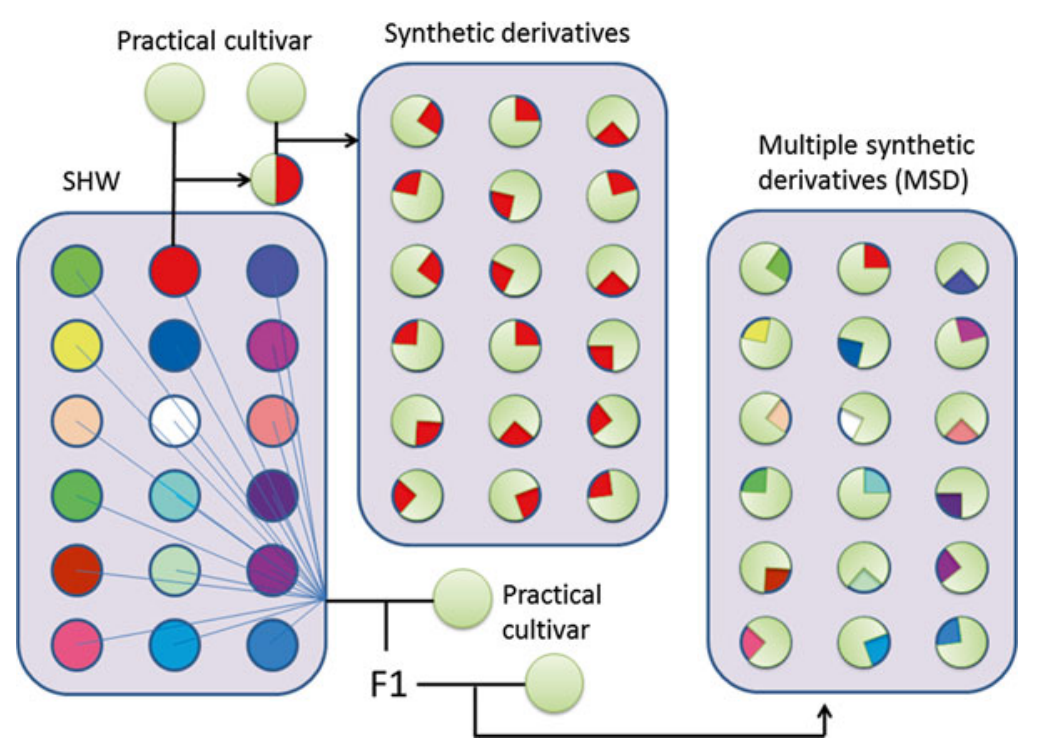

Fig. 25.3 Production of a multiple synthetic derivative population containing the genetic diversity of Ae. tauschii. SHW, synthetic hexaploid wheat

robust appearance with long and tough spikes, very different from the morphology of common wheat cultivars.

In general, an SHW line is crossed twice with a common wheat line and the resulting derivative populations used as initial selection materials for breeding (Fig. 25.3). In this protocol, however, only one Ae. tauschii accession can provide the genes of the derivative population. Thus, to obtain material populations 
containing the wide genetic diversity of Ae. tauschii, we crossed each SHW with 'Norin 61' (N61), a Japanese standard cultivar that has wide adaptability, and Sephadak Ishkashim W (SIW), a landrace from the Ishkashim region of Tajikistan and Afghanistan. As a result, we obtained 43 and $40 \mathrm{~F}_{1}$ hybrids, respectively. The $\mathrm{F}_{1}$ progenies were then backcrossed, resulting in 1,893 and 2,546 $\mathrm{BC}_{1} \mathrm{~F}_{1}$ plants, respectively. These plants were then self-pollinated to produce $\mathrm{BC}_{1} \mathrm{~F}_{2}$ seeds. Ten seeds from $10 \mathrm{BC}_{1} \mathrm{~F}_{1} \mathrm{~s}$ from each line were mixed, resulting in seed-stock populations containing the genetic diversity of Ae. tauschii in the N61 and SIW backgrounds (Fig. 25.3). Plants from these mixed-seed stock populations showed a diverse morphology, allowing concise evaluation of the traits associated with abiotic stress tolerance. Genomes of more than 40 accessions of Ae. tauschii contributed to these populations and as a result, it is believed they contain most of the genetic diversity of Ae. tauschii within the common wheat genetic background. Furthermore, by obtaining seeds derived from different crosses mixed in one population, the handling of materials for screening was made easier. In fact, the $\mathrm{BC}_{1} \mathrm{~F}_{1}$ plants showed a different response to drought stress.

In addition to the above, we also crossed T. durum cv. 'Miki 3', a leading cultivar from ICARDA provided by Dr. M. Nachit, with nine accessions of wild tetraploid Emmer wheat, $T$. dicoccoides. The $\mathrm{F}_{1}$ plants were then backcrossed with Miki three, giving a population consisting of $236 \mathrm{BC}_{1} \mathrm{~F}_{1}$ plants, all showing a diverse morphology (although some with brittle spikes). The $\mathrm{BC}_{1} \mathrm{~F}_{1}$ plants were then selfed and the seeds $\left(\mathrm{BC}_{1} \mathrm{~F}_{2}\right)$ were mixed to produce a seed stock population.

We collectively named these hexaploid and tetraploid stock populations 'multiple derivative lines' (MDLs), and specifically the hexaploid populations were designated 'multiple synthetic derivatives'. MDL populations can be used for various breeding purposes, because they show potentially valuable traits derived from diverse wild species in the cultivar-derived genetic background. Moreover, the morphological similarity of each plant in the population makes the selection of complex traits feasible.

In general, the phenotype of any individual is influenced by genotype, environmental factors, and the interaction between the two. Because the genotypeenvironment interaction is thought to play a significant role in the expression of drought tolerance in wheat, finding genotypes well adapted to dryland environments is usually a difficult task, especially under conditions that fluctuate greatly from year to year. As a result, common modern breeding practices; that is, determining the "best" genotype that outperforms in a set of material accessions under given conditions, may not be useful in wheat drought tolerance breeding, since the "best" drought-tolerant genotype may differ depending on the cropping year and location. Traditionally, wheat farmers cope with unpredictable environmental changes by intentionally cultivating various landraces in the mix (Matsuoka et al. 2008). Accordingly, one alternative approach in wheat drought tolerance breeding would be to produce a genetically diverse material population and perform sowingharvesting cycles at a local site for several years. This way, an optimal genetic structure could be realized in the descendant population through the natural process of local adaptation. We believe the MDLs could provide useful materials for wheat 
drought tolerance breeding using such an approach. By monitoring allele frequency transition in a MDL population under cultivation using molecular markers, the genetic mechanisms that underlie the local adaption could subsequently be studied in depth. In principle, the MDL-based approach relies on the natural adaption process that wheat landraces must have undergone. For this reason, the above approach may allow development of a novel field of breeding science we call 'evolutionary breeding'. Furthermore, the MDL-based approach would also provide a great opportunity for participatory breeding of drought-tolerant wheat.

Open Access This chapter is distributed under the terms of the Creative Commons Attribution Noncommercial License, which permits any noncommercial use, distribution, and reproduction in any medium, provided the original author(s) and source are credited.

\section{References}

Matsuoka Y, Aghaei MJ, Abbasi MR et al (2008) Durum wheat cultivation associated with Aegilops tauschii in northern Iran. Genet Resour Crop Evol 55:861-868

Matsuoka Y, Nasuda S, Ashida Y et al (2013) Genetic basis for spontaneous hybrid genome doubling during allopolyploid speciation of common wheat shown by natural variation analyses of the paternal species. PLoS One 8:e68310

Sohail Q, Inoue T, Tanaka H et al (2011) Applicability of Aegilops tauschii drought tolerance traits to breeding of hexaploid wheat. Breed Sci 61:347-357

Sohail Q, Shehzad T, Kilian A et al (2012) Development of diversity array technology (DArT) markers for assessment of population structure and diversity in Aegilops tauschii. Breed Sci $62: 38-45$ 